\title{
Fuzzy Structural Analysis of a Tuned Mass Damper Subject to Random Vibration
}

\author{
Giuseppe Carlo Marano, Emiliano Morrone, Giuseppe Quaranta, and Francesco Trentadue \\ Dipartimento di Ingegneria dell'Ambiente e per lo Sviluppo Sostenibile (DIASS), Politecnico di Bari, Viale del Turismo 8, \\ 74100 Taranto, Italy \\ Correspondence should be addressed to Giuseppe Carlo Marano, gmarano@poliba.it
}

Received 1 October 2008; Accepted 27 November 2008

Recommended by Miguel Ayala Botto

The uncertainty is a typical feature of each human activity since the greatest part of the information is always affected by a sure level of scattering. Different methodologies which deal with the uncertainty of the real problems exist. The principal aim of this paper is to present an innovative hybrid approach which combines fuzzy and stochastic theories in facing the structural analysis of a tuned mass damper subject to a dynamic random load, modelled by a modulated filtered white noise. In this work the parameters involved in the structural analysis will be considered uncertain and supposed fuzzy sets to take into account the effects of lexical and informal uncertainties which cannot be studied in a probabilistic way. The system analysis is conducted by means of $\alpha$-level optimization technique. Successively, a numerical example is presented to show the effectiveness of the proposed procedure. Moreover, a sensitivity analysis is performed to expose the variation of the structural response membership function considering different input values. Finally, a comparison between the response nominal value and the fuzzificated one is proposed to obtain a structural amplification factor.

Copyright (c) 2008 Giuseppe Carlo Marano et al. This is an open access article distributed under the Creative Commons Attribution License, which permits unrestricted use, distribution, and reproduction in any medium, provided the original work is properly cited.

\section{INTRODUCTION}

The limitation of the effects of the vibrations due to the environmental dynamic loads is a very important matter in structural engineering. In this field, many different strategies have been proposed, especially with regard to the problems of the random vibrations induced by natural or artificial loads, as those due to the earthquakes, wind pressure, traffic vibrations, sea waves, and so on. Generally, in literature, four groups of control systems are distinguished: active, hybrid, semiactive, and passive. Among these, the passive systems are the most unsophisticated and cheapest. These systems are characterized by the fact that they completely manage and dissipate energy in a mechanical way [1]. For this reason, they have several important advantages: the systems are purely mechanical, often simplistic, and do not require external power or device that is an important source of robustness. The passive systems have been used successfully in many real cases. Among numerous passive control available devices, the tuned mass damper (TMD) is frequently adopted both in mechanical systems and in civil structures, in new constructions as well as in the retrofit of existing buildings. In general, the purpose of installing a TMD is to guarantee a suitable level of protection in the primary structure to assure an adequate safety level, both for the structure for its contents regarding a defined limit state. Moreover, TMD is introduced to reduce the discomfort to occupants and/or to limit the damage of the equipments in particular into high-rise buildings, especially when moderate (and frequent) seismic loads are taken into account in the design process. In fact, recent earthquakes showed that in the equipments and in the contents of buildings the damage can have large economic consequences. For instance, in high-rise buildings, in several displacement of sensitive nonstructural systems (suspend ceilings, light fixture, fire suppression piping systems, computer systems, emergency power generation systems, elevators, etc.), a localized damage can affect the functionality of a large portion of the edifice.

Therefore, the structural seismic design should be applied not only to guarantee the life safety and prevent 
structural collapse, but also to ensure a given level of performance. Afterwards, the structural behavior is difficult to analyze because a lot of uncertainty sources are involved; for this reason, many adequate approaches have been developed. For instance, in the probabilistic field, the methodology based on stochastic dynamic analysis is widely adopted. In fact, Rüdinger [2] investigated the response of a nonlinear TMD subject to a stationary white noise obtaining some optimal mechanical parameters. Marano et al. [3-5] proposed another interesting approach based on a procedure in which a single linear TMD problem is treated and on the fact that the earthquake can be represented by a stationary filtered stochastic process. In their work, the main goal is to minimize the maximum of the dimensionless peak of displacement of the protected system in respect of the unprotected one. The latter papers and other examples proposed in literature $[6,7]$ show the arising necessity of the designers to investigate in probabilistic terms the structural response in order to face properly the uncertainties which affect the input load. On the other hand, in a lot of real cases, the probabilistic structural analysis is poorly suitable and this is due to fact that a relevant number of parameters cannot be assumed as random variables. For example, Takewaki and Ben-Haim [8] adopted some info-gap models for load and some model uncertainty parameters in stout design to achieve the robustness function for single degree of freedom (SDOF) and multidegree of freedom (MDOF) structures. In the field of nonprobabilistic uncertainties, the theory based on the concept of fuzzy sets [9] seems to be the most appropriate because of its capability of taking into account the so-called lexical and informal uncertainties which affect the structural system.

In this paper, a new methodology is proposed to analyze the response of a TMD subject to a nonstationary filtered white noise using a hybrid approach based on the fuzzy and stochastic theories. Many authors dealt with fuzzy theory in analyzing structural response [10-12] but they did not consider that also the inherent stochastic nature of input processes can be affected by lexical and formal uncertainties that should be treated in a fuzzy perspective. As a consequence, in this work, all model and data parameters are assumed as fuzzy variables. So, the membership function (MF) of the maximum standard deviation displacement of the protected structure is gotten by means of the $\alpha$-level optimization method. Moreover, an analysis of design sensitivity is executed to establish the effects of the most important uncertain parameters in evaluation of the structural behavior. Finally, a fuzzy-based indicator is introduced to assess how the nominal value of the structural performance is amplified performing a fuzzy-based analysis.

\section{THE ROLE OF THE NONPROBABILISTIC UNCERTAINTIES}

It should be observed that not all the unsure variables can be treated following the standard probabilistic approach. It is not the most adequate method to describe the uncertainty for the most part of the realistic conditions and its use could be sometimes extremely poor for real problems.

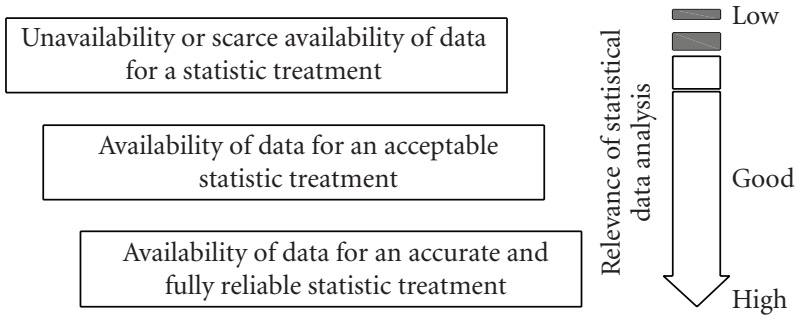

FIGURE 1: The relevance of statistical data analysis.

In particular, nonprobabilistic uncertainties deal with the so-called "epistemic uncertainty" that is seldom agreeable to probabilistic characterization. A typical classification of epistemic uncertainty can be as follows.

\section{Idealization uncertainty}

The main goal to perform a reliable structural analysis is the idealization of reality into a mathematical boundary value problem. This entails continuum mechanics assumptions that provide mathematical equations for equilibrium, kinematics, and constitutive behavior, as well as boundary conditions. Epistemic uncertainty is thereby introduced due to imperfect model form and/or missing parameters.

\section{Complexity uncertainty}

The parameters of an idealized boundary value problem are commonly determined by laboratory tests of small specimens. A useful example of this is furnished by stress paths in a beam-column connection or the bonding between concrete and reinforcement which are complicated phenomena whose models and data are limited. Hence, the estimates from simplified laboratory tests carry uncertainty.

\section{Statistical uncertainty}

Parameters such as material stiffness and strength are assessed based on available data. Uncertainty arises when the number of observations is limited, which is frequently the case. This leads to uncertainty in the distribution parameters of the random variables and is referred to as statistical uncertainty. Moreover, the observations of a variable rarely represent it perfectly and as result often a bias in data recorded is automatically generated.

From above definitions, it can be observed that nonprobabilistic uncertainties are often present when only few data about one or more models and the structural parameters are available or when the investigation of the properties and of the load conditions of the mechanical and geometrical systems is economically inconvenient or impossible. For example, this happens in the historical constructions. A further not negligible theme is the significance of the "expert opinion" in some phases of the design process because of the necessity and the importance of a specific technical point of view. These observations could be summarized in Figure 1. 
In this field, in the last few years new methodologies has been proposed. The most interesting one is based on the theory of fuzzy sets [9]. Savoia [13] developed this hypothesis and defined an equivalent and compatible probability distribution for fuzzy available data.

Moreover, a fuzzy randomness application presented by Möller et al. [14] and a fuzzy Monte Carlo simulation with fuzzy adaptive importance sampling method are performed to estimate the time-dependent fuzzy failure probability.

Finally, the linear time invariant dynamical system has been investigated by $\mathrm{Hu}$ et al. [15] considering that input signals are second order fuzzy stochastic process.

\section{FUZZY STRUCTURAL ANALYSIS OF TUNED MASS DAMPER}

\subsection{The fuzzy-based description of uncertainties}

The variables on which lexical or informal uncertainty encumbers could be treated by means of fuzzy sets theory. Its greatest advantage is the possibility of a gradual assessment of the grade of membership of the elements in relation to a generic set. This continuum degree of membership is described by the so-called membership function (MF). Initially, in fuzzy structural analysis, one of the problems is to acquire the MF for each fuzzy input. In this phase, the main goal is to capture the meanings of the nonprobabilistic parameters. This problem belongs to the area of "knowledge acquisition." Generally, it is possible to assert that for the so-called fuzzification, a unitary approach does not exist. Different procedures could be adopted for each situation relatively to the available information. In literature, it is useful to classify the methods for constructing MF as direct or indirect and with single or multiple experts. In our work, we assume that the MF is known for each fuzzy variable. In other words, we suppose that a specific and adequate knowledge acquisition approach has been performed. For a TMD, different sources of uncertainties can be found in both data and model. In detail, in our work, we suppose that each variable can be modelled by means of a fuzzy number. At a first analysis, this assumption can appear extreme but we should observe that in any real situation it is impossible to consider a parameter as a deterministic datum. In fact, the uncertainty can be reduced but never entirely eliminated. In particular, in the greatest part of the circumstances, the structural parameters are known with extreme inaccuracy. This is especially true for structural damping. Energy absorption and dissipation as well as the structural ductility can be burdened by a notable indeterminateness. Furthermore, the model parameters adopted to represent a random vibration can be considered as nonprobabilistic variables. In the dynamic analysis, a typical evidence of this can be the valuation of the soil type; it can be stiff, medium, or soft. This consideration is imputable to the lack of information for a reliable statistic analysis. Finally, it is possible to notice a great variability (often in regional and subregional scales ) of these parameters and in this case the expert opinion can play a fundamental role in the assessment of their values and intervals of confidence. For what concerns the TMD, we can affirm that it is an industrial product with a defect consisting in its mechanical parameters; in fact, for them, it is often necessary to consider a certain error in the evaluation. Besides, these devices are frequently furnished with datasheets in which some information on the variability of these data is indicated.

\subsection{The $\alpha$-level optimization method}

Once these fuzzy inputs are known with their respective MF, the principal aim of a fuzzy structural analysis is to "map" both fuzzy inputs and model characteristics into a response which is also fuzzy. The function (or algorithm) realizing this mapping is a deterministic one and is called mapping operator. In literature, two methodologies exist to obtain the output MF. A first procedure is based on the concept of the extension principle, but the most effective approach is found on the discretization of the fuzzy sets, and so on the consequent application of the $\alpha$-level optimization [16]. Let us consider some generic $n$ fuzzy input variables:

$$
\tilde{Y}_{i}=\left\{\left(y_{i}, \mu\left(y_{i}\right)\right) \mid y_{i} \in \mathbf{Y}_{i}\right\} \quad i=1, \ldots, n,
$$

where $\mu\left(y_{i}\right)$ and $\mathbf{Y}_{i}$ are, respectively, the MF and the fundamental set of the uncertain one $\widetilde{Y}_{i}$ (or fuzzy value $\tilde{y}_{i}$ ). Then, for each fuzzy variable, the crisp sets

$$
Y_{i}^{\alpha_{k}}=\left\{y_{i} \in \mathbf{Y}_{i} \mid \mu\left(y_{i}\right) \geq \alpha_{k}\right\} \quad i=1, \ldots, n
$$

can be extracted for each $\alpha$-cut $\alpha_{k} \in[0,1]$, and they are denoted as $\alpha$-level sets. Subsequently, the Cartesian product between the $\alpha$-level sets defines the input $n$-dimensional crisp subspace:

$$
\underline{Y}^{\alpha_{k}}=\left\{Y_{1}^{\alpha_{k}} \otimes Y_{2}^{\alpha_{k}} \cdots \otimes Y_{i}^{\alpha_{k}} \cdots \otimes Y_{n}^{\alpha_{k}}\right\} .
$$

If we denote with $f(\cdot)$ the deterministic mapping operator, the following relation holds:

$$
\begin{aligned}
& \forall u \in\left[\min _{\underline{Y}^{\alpha_{k}}} f\left(y_{1}, \ldots, y_{i}, \ldots y_{n}\right), \max _{{\underline{Y^{\alpha}}}^{\alpha_{k}}} f\left(y_{1}, \ldots, y_{i}, \ldots y_{n}\right)\right] \\
& \Longrightarrow \mu(u) \geq \alpha_{k} .
\end{aligned}
$$

Therefore,

$$
\begin{array}{r}
U^{\alpha_{k}}=\left\{u \in \left[\min _{\underline{Y}^{\alpha_{k}}} f\left(y_{1}, \ldots, y_{i}, \ldots y_{n}\right),\right.\right. \\
\left.\left.\max _{{\underline{Y^{\alpha}}}^{\alpha_{k}}} f\left(y_{1}, \ldots, y_{i}, \ldots y_{n}\right)\right]\right\}
\end{array}
$$

is the output $\alpha$-level set. Afterwards, the output fuzzy set is given by

$$
\tilde{U}_{i}=\bigcup_{\alpha \in[0,1]} U^{\alpha_{k}}=\left\{\left(u_{i}, \mu\left(u_{i}\right)\right) \mid y_{i} \in \mathbf{U}_{i}\right\} \quad i=1, \ldots, n .
$$

This procedure is shown in Figure 2 for $n=2$ and for a generic $\alpha$-cut. 


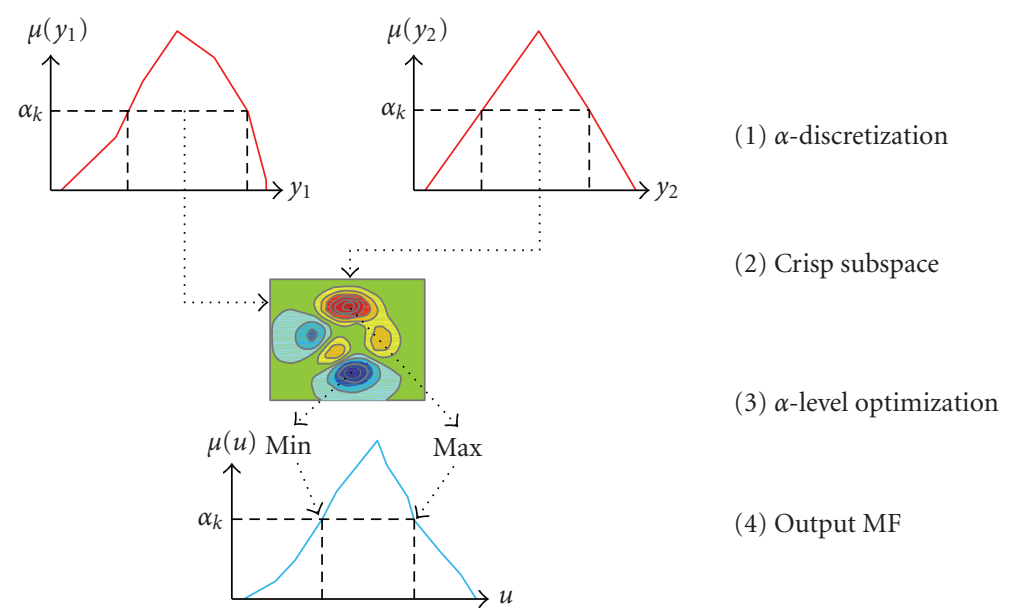

FIgURE 2: The $\alpha$-level optimization.

Formally, this procedure is defined by a double optimization problem for each $\alpha$-cut, as it is expressed in (4). When the mapping operator is nonmonotonic with reference to all the fuzzy input variables (e.g., as it is illustrated in Figure 2), the minimum and maximum values cannot be achieved at the vertices of the crisp subspace (3). In this condition, a numerical algorithm must be adopted and so the procedure can become even more complex.

\section{DYNAMIC ANALYSIS OF TMD UNDER CONSIDERATION ON FUZZINESS}

The mechanical system is made up by a principal mass linked through a spring and a damper to another secondary mass provided with a spring and a damper also. The latter protects the structure from high-intensity vibration because it oscillates with the same frequency of the main structural system but with a phase shift. The main structural system is an SDoF because in the principal application of civil engineering we can refer to the first vibration mode of a generic MDoF system [17]. The goal of the investigation is to get the membership function of the peak displacement response of the described system subject to a nonstationary seismic excitation. We can reach this aim by using the $\alpha$-level optimization procedure when all the involved parameters are fuzzy variables.

The choice of this quantity is very interesting from an engineering point of view because it is a fundamental criterion in new design technologies as performance- and displacement-based designs. Many authors [16] affirmed that the obtained algorithm is the best. It is used in the optimization as an evolutionary one because it is able to guarantee a robust optimization technique independent from the OF type and capable of finding some global optima. For these reasons, the use of a genetic algorithm seems the best preference to adopt. In all conducted simulations, a standard MATLAB genetic algorithm has been used [18].

The dynamic mechanical model and its properties are reported in Figure 3. Hence, for the sake of simplicity, the symbol $\alpha_{k}$ is omitted for each fuzzy parameter.
Being

$$
\mu_{T}=\bigcup_{\alpha \in[0,1]} \mu_{T}^{\alpha_{k}}=\bigcup_{\alpha \in[0,1]}\left(\frac{m_{T}^{\alpha_{k}}}{m_{s}^{\alpha_{k}}}\right)
$$

If $\underline{D}^{\alpha_{k}}$ denotes the input 8-dimensional crisp subspace, given by the following Cartesian product:

$$
\underline{D}_{\alpha_{k}}=\left[\Omega_{s}^{\alpha_{k}} \otimes \Xi_{s}^{\alpha_{k}} \otimes \Omega_{T}^{\alpha_{k}} \otimes \Xi_{T}^{\alpha_{k}} \otimes M_{T}^{\alpha_{k}} \otimes \Omega_{f}^{\alpha_{k}} \otimes \Xi_{f}^{\alpha_{k}} \otimes \ddot{X}_{g}^{\max , \alpha_{k}}\right],
$$

the standard deviation of the response is given by

$$
\begin{array}{r}
\Sigma^{\alpha_{k}}=\left\{\sigma _ { X } ^ { \operatorname { m a x } } \in \left[\min _{\underline{D}^{\alpha_{k}}} f\left(\omega_{S}, \xi_{s}, \omega_{T}, \xi_{T}, \omega_{f}, \xi_{f}, \mu_{T}, \ddot{X}_{g}^{\max }\right),\right.\right. \\
\left.\left.\max _{\underline{D}^{\alpha_{k}}} f\left(\omega_{S}, \xi_{s}, \omega_{T}, \xi_{T}, \omega_{f}, \xi_{f}, \mu_{T}, \ddot{X}_{g}^{\max }\right)\right]\right\},
\end{array}
$$

where in this particular case the mapping operator is provided by

$$
\sigma_{X}^{\max }=f\left(\omega_{S}, \xi_{s}, \omega_{T}, \xi_{T}, \omega_{f}, \xi_{f}, \mu_{T}, \ddot{X}_{g}^{\max }\right),
$$

so that, the fuzzy output set is calculated by

$$
\tilde{\Sigma}=\bigcup_{\alpha \in[0,1]} \Sigma^{\alpha_{k}}=\left\{\left(\sigma_{X}^{\max }, \mu\left(\sigma_{X}^{\max }\right) \mid \sigma_{X}^{\max } \in \Sigma\right\} .\right.
$$

Our purpose is to find $\tilde{\Sigma}$ by the mapping operator defined in (10). We can say about the input process that the response evaluation must be based on a stochastic approach because of its random nature. As commonly known, the ground accelerograms are often modelled as a zero-mean stochastic nonstationary process. This assumption is able to consider the intrinsic probabilistic nature of earthquakes in the real way. Moreover, a suitable description with no constant contents both in amplitude and in frequency could be taken into account. A widely used stochastic approach is the one proposed by Tajimi [19], based on a linear second-order filter 


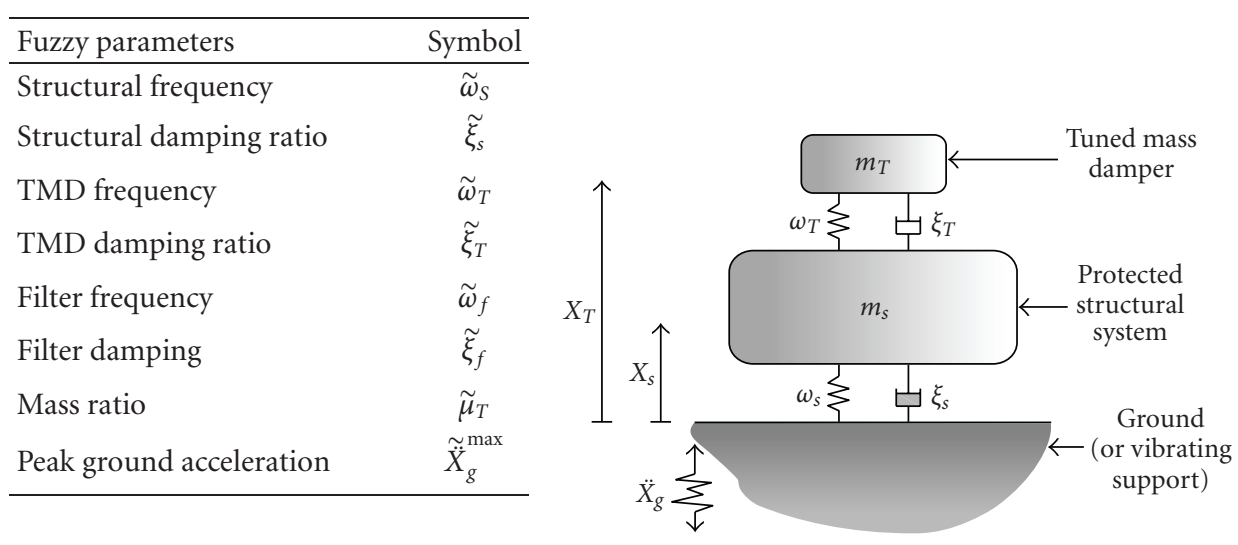

Figure 3: The structural dynamical model.

applied to a stationary white noise. Therefore, the ground acceleration $\ddot{X}_{g}$ is calculated by

$$
\begin{gathered}
\ddot{X}_{g}(t)=\ddot{X}_{f}(t)+\varphi(t) W(t), \\
\ddot{X}_{f}(t)+2 \xi_{f} \omega_{f} \dot{X}_{f}(t)+\omega_{f}^{2} X_{f}(t)=-\varphi(t) W(t),
\end{gathered}
$$

where $X_{f}(t)$ is the response of the Kanai-Tajimi filter, having frequency $\omega_{f}$, damping coefficient $\xi_{f}$, eventually time depending, and white noise $W(t)$ whose constant bilateral power spectral density (PSD) function is $S_{0}$. It is related to the peak ground acceleration (PGA) $\ddot{X}_{g}^{\max }$ by means of relation [20]

$$
S_{0}=\frac{0.222}{\pi} \frac{\xi_{f}\left(\ddot{X}_{g}^{\max }\right)^{2}}{\omega_{f}\left(1+4 \xi_{f}^{2}\right)} .
$$

No stationary characteristic is introduced by the deterministic temporal modulation function. It controls the intensity variation without changing the contents of the earthquake frequency. Moreover, different modulation functions have been proposed in literature with the aim of best accordance with the real-time evolution of accelerograms. In our work, we adopt the modulation function proposed by Jennings et al. [21] as follows:

$$
\varphi(t)= \begin{cases}\left(\frac{t}{t_{1}}\right)^{2}, & t<t_{1}, \\ 1, & t_{1} \leq t \leq t_{2} \\ e^{-\beta\left(t-t_{2}\right)}, & t>t_{2} .\end{cases}
$$

Making the assumption that the system is linear and the input is Gaussian zero-mean process, the knowledge of the covariance matrix can fully characterize the space state response statistics, as a result of the fact that the response is Gaussian zero-mean too. Then, the probabilistic analysis is performed by solving the well-known Lyapunov matrix differential equation.
Therefore, let us consider the state vector $\bar{Z}$ :

$$
\bar{Z}(t)=\left[\begin{array}{c}
X_{s}(t) \\
X_{T}(t) \\
X_{f}(t) \\
\dot{X}_{s}(t) \\
\dot{X}_{T}(t) \\
\dot{X}_{f}(t)
\end{array}\right],
$$

the motion equation of the structure plus filter in the space state is

$$
\bar{Z}(t)=\mathbf{A} \bar{Z}(t)+\bar{F}(t)
$$

where

$$
\bar{F}(t)=\left(\begin{array}{c}
0 \\
0 \\
0 \\
0 \\
0 \\
-\varphi(t) W(t)
\end{array}\right)
$$

is the forcing vector, and

$$
\mathbf{A}=\left(\begin{array}{cccccc}
0 & 0 & 0 & 1 & 0 & 0 \\
0 & 0 & 0 & 0 & 1 & 0 \\
0 & 0 & 0 & 0 & 0 & 1 \\
\mathcal{U} & \mu_{T} \omega_{T}^{2} & \omega_{f}^{2} & \mathcal{P} & 2 \mu_{T} \xi_{T} \omega_{T} & 2 \omega_{f} \xi_{f} \\
\omega_{T}^{2} & -\omega_{T}^{2} & \omega_{f}^{2} & 2 \xi_{T} \omega_{T} & -2 \mu_{T} \xi_{T} \omega_{T} & 2 \omega_{f} \xi_{f} \\
0 & 0 & -\omega_{f}^{2} & 0 & 0 & -2 \omega_{f} \xi_{f}
\end{array}\right)
$$

(where $\mathcal{U}$ denotes $-\omega_{S}^{2}-\mu_{T} \omega_{T}^{2}$ and $\mathcal{P}$ denotes $-2 \xi_{S} \omega_{S}-$ $\left.2 \mu_{T} \xi_{T} \omega_{T}\right)$ represents the system matrix.

So starting from the state equation (16), the covariance analysis is performed by solving the Lyapunov matrix differential equation:

$$
\dot{\mathbf{R}}_{\bar{Z} \bar{Z}}(t)=\mathbf{A R}_{\bar{Z} \bar{Z}}(t)+\mathbf{R}_{\bar{Z} \bar{Z}}(t) \mathbf{A}^{T}+\mathbf{B}(t) .
$$

In (19),

$$
\mathbf{R}_{\bar{Z} \bar{Z}}(t)=\mathbf{E}\left[\bar{Z} \bar{Z}^{T}\right]
$$


TABLE 1: Fuzzy input for the numerical example.

\begin{tabular}{lc}
\hline Fuzzy variables & Values \\
\hline$\widetilde{\omega}_{f}$ & $\langle 10,15,20\rangle[\mathrm{rad} / \mathrm{s}]$ \\
$\widetilde{\xi}_{f}$ & $\langle 0.35,0.45,0.65\rangle[\mathrm{ad}]$ \\
$\widetilde{\omega}_{s}$ & $\langle 10,12,15\rangle[\mathrm{rad} / \mathrm{s}]$ \\
$\widetilde{\xi}_{s}$ & $\langle 0.01,0.02,0.05\rangle[\mathrm{ad}]$ \\
$\widetilde{X}_{g}$ & $\langle 0.15,0.20,0.25\rangle[\mathrm{g}]$ \\
$\tilde{\mu}_{\mathrm{TMD}}$ & $\langle 0.04,0.08,0.12\rangle[\mathrm{ad}]$ \\
$\widetilde{\omega}_{\mathrm{TMD}}$ & $\langle 9,12,14\rangle[\mathrm{rad} / \mathrm{s}]$ \\
$\widetilde{\xi}_{\mathrm{TMD}}$ & $\langle 0.08,0.12,0.15\rangle[\mathrm{ad}]$ \\
\hline
\end{tabular}

is the covariance matrix $(\mathbf{E}[\cdot]$ denotes the mathematical expectation), while $\mathbf{B}(t)$ contains all elements equal to zero except the last one that holds $2 \pi S_{0} \varphi(t)^{2}$.

Afterwards, if we consider the time interval $[0, T]$, the relevant quantity can be obtainable by

$$
\begin{aligned}
\sigma_{X}^{\max } & =f\left(\omega_{S}, \xi_{s}, \omega_{T}, \xi_{T}, \omega_{f}, \xi_{f}, \mu_{T}, \ddot{X}_{g}^{\max }\right) \\
& =\sqrt{\max _{t \in[0, T]} \mathbf{R}_{\bar{Z} \bar{Z}}(1,1)} .
\end{aligned}
$$

For a generic $\alpha_{k}$, expression (9) becomes

$$
\begin{aligned}
\Sigma^{\alpha_{k}}=\left\{\sigma _ { X } ^ { \operatorname { m a x } } \in \left[\min _{\underline{D}^{\alpha_{k}}} \sqrt{\max _{t \in[0, T]} \mathrm{R}_{\bar{Z} \bar{Z}}(1,1)},\right.\right. \\
\left.\left.\max _{\underline{D}^{\alpha_{k}}} \sqrt{\max _{t \in[0, T]} \mathrm{R}_{\bar{Z} \bar{Z}}(1,1)}\right]\right\}^{\alpha_{k}} .
\end{aligned}
$$

\section{NUMERICAL EXAMPLES}

In the present work, several numerical simulations have been carried out to assess the proposed methodology and to conduct a sensitivity analysis. For all the simulations, all the membership functions of fuzzy variables are supposed to be triangular and the amplitude of the modulation function is 7 seconds in (14), while $\beta=0.4$. The $\alpha$-cut step is fixed to 0.2 .

The first analysis has been conducted to calculate the membership function of $\sigma_{X}^{\max }$ assigned the values reported in Table 1.

About the filter frequency and the damping ratio, the support describes the values that a stiff soil can assume with a medium assessment given by the core. In practical applications or in research works, the estimation of the soil type is affected by a remarkable degree of uncertainty which can be difficult quantified. Moreover, in the prefilters technique which is an extensively adopted methodology to model the ground in dynamic structural analysis, the evaluation of the values of the parameters is often purely conventional and there are no physical reasons to justify them once they have been defined. In fact, in literature, there are many authors' proposals about this issue [22] but none of them seems to be coherent to design code spectra or to scale really seismic simulations. For what concerns the support assigned to the structural frequency and damping ratio, we can say that the values are typical of

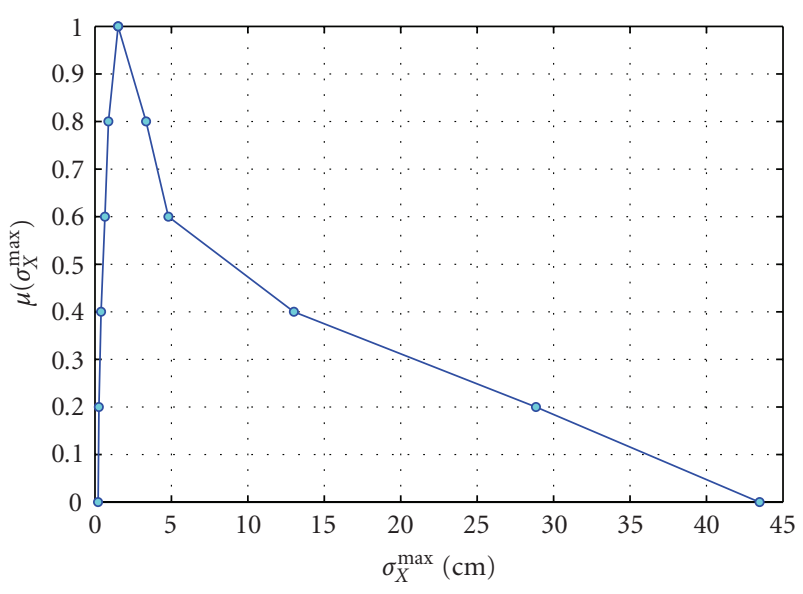

FIGURE 4: The maximum displacement standard deviation MF.

civil buildings. In practical applications, their assessment is difficult because there are few experimental data about them. The best analysis which can be performed is a postevent one but it is worthless. As a consequence, the properties of the real structure are often subject to the expert judgments which are evidently affected by informal and lexical uncertainties. Another important aspect is the ground acceleration support which represents the values that can be assumed in evaluating a site classified as B by the Eurocode 8. It is well known that the hazard maps expressed in terms of peak ground acceleration or in general by means of an intensity factor are notably simplified; in fact, real analysis on the behavior of the seismic waves show that they change both their characteristics and their energy content going through each soil layer. As a consequence, the spatial variation of the peak ground acceleration is a significant source of uncertainty. The values of the TMD parameters are regularly presented in the datasheets of the producers but for each of them a certain degree of tolerance has to be considered. Simulation outcome is shown in Figure 4. Examining Figure 4, several considerations can be made distinguishing several values of degree of membership.

If $\mu\left(\sigma_{X}^{\max }\right)=60 \div 100 \%$, the output values are evidently concentrated near the core. So if we assume (as widely adopted in current literature) that the core corresponds to the probabilistic mean value, this denotes that the confidence interval of structural response estimation is near the one that can be obtained by performing a purely probabilistic analysis.

If $\mu\left(\sigma_{X}^{\max }\right)=30 \div 60 \%$ (so the degree of membership is considerably lower), it can be noticed that the distance of the output values from the core is increasing. In practical, occurrences these values of response degree of membership, which cannot be usually excluded, lead to a lower assessment of the structural performance and to an overestimation of the structural reliability.

If $\mu\left(\sigma_{X}^{\max }\right)=0 \div 30 \%$, the latter trend continues; in fact, the scattering from the core is very huge. However, we have to mention that this degree of membership can rarely occur in real applications. 
TABle 2: Different adopted structural frequency membership functions.

\begin{tabular}{lc}
\hline Fuzzy variables & Values \\
\hline$\diamond \widetilde{\omega}_{S}$ & $\langle 10,10.50,15\rangle[\mathrm{rad} / \mathrm{s}]$ \\
$\circ \widetilde{\omega}_{S}$ & $\langle 10,14.50,15\rangle[\mathrm{rad} / \mathrm{s}]$ \\
\hline
\end{tabular}

TABle 3: Different adopted structural frequency membership functions.

\begin{tabular}{lc}
\hline Fuzzy variables & Values \\
\hline$\diamond \widetilde{\xi}_{S}$ & $\langle 0.01,0.0125,0.05\rangle[\mathrm{ad}]$ \\
$\circ \widetilde{\xi}_{S}$ & $\langle 0.01,0.0475,0.05\rangle[\mathrm{ad}]$ \\
\hline
\end{tabular}

Once the MF of response has been obtained, a sensitivity analysis has been conducted to affirm how a scattering of both structural frequency and damping can affect its behavior. To complete these investigations, two types of MF for $\widetilde{\omega}_{s}$ and $\tilde{\xi}_{s}$ are considered. One with a core moved to the left of MF and one moved to the right. The numerical values are represented in Tables 2 and 3, respectively, for $\widetilde{\omega}_{s}$ and $\tilde{\xi}_{s}$.

Figures 5 and 6 show the result of the conducted sensitivity analysis with respect to $\widetilde{\omega}_{s}$ and $\tilde{\xi}_{s}$. A first observation is evident. Examining the output MF in the first case (blue line), we can notice that the core has a value which tends to be zero because moving $\omega_{s}$ to the right is equivalent to supposing that the system is very stiff, the same holds for $\widetilde{\xi}_{s}$, moving this parameter MF implies to ensure a high-structural dissipation capacity. In addition, we have to mention that the blue and the red lines are almost superposed for what concerns the left branch of the MFs. Moreover, in Figure 6 the cores of the two MFs are very near to each other. However, in both cases the difference of structural response tends to be against safety considering only the right branches of the MFs. A variation in the structural frequency causes the highest scattering in the structural response.

Finally, a fuzzy indicator has been derived to estimate how much the defuzzificated output MF is far from the nominal output value $\sigma_{c X}^{\max }$ at $\mu\left(\sigma_{X}^{\max }\right)=1$. The defuzzificated variable is gotten evaluating the center of gravity of the fuzzy number $\widetilde{\Sigma}$ :

$$
G(\widetilde{\Sigma})=\frac{\int_{\sigma_{l X}^{\max }}^{\sigma_{u X}^{\max }} \sigma_{X} \mu\left(\sigma_{X}\right) \mathrm{d} \sigma_{X}}{\int_{\sigma_{l X}^{\max }}^{\sigma_{u X}^{\max }} \mu\left(\sigma_{X}\right) \mathrm{d} \sigma_{X}},
$$

where in (23) the subscripts " $u, 1$, and $c$," respectively, stay for upper, lower, and core. The meaning of these subscripts is graphically represented in Figure 7 in order to clarify how structural amplification factor is obtained.

The above-mentioned fuzzy indicator, named, structural response amplification factor is given by

$$
\gamma=\frac{G(\tilde{\Sigma})}{\sigma_{c X}^{\max }}
$$

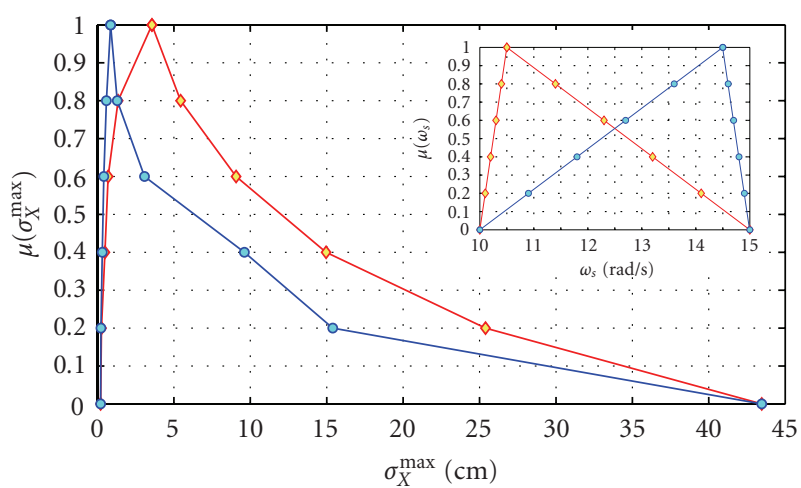

FIGURE 5: The sensitivity analysis on the structural frequency.

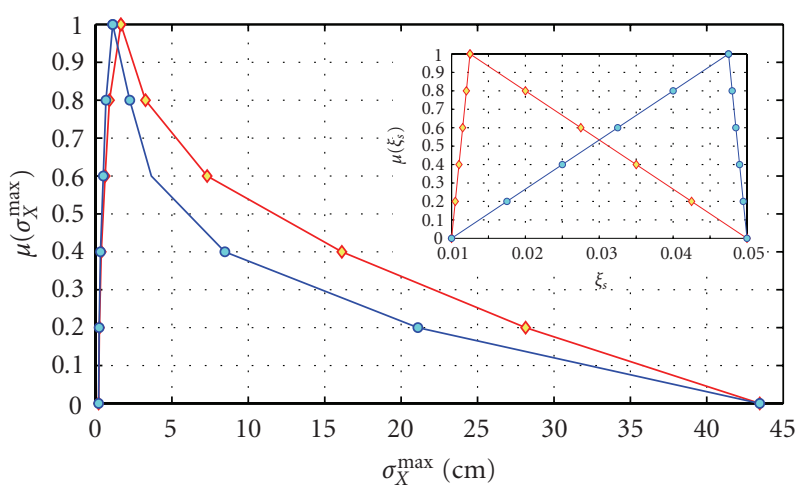

FIGURE 6: The sensitivity analysis on the structural damping.

Applying (23) to the MF given by Figure 4, we obtain a value of the centroid of $13.60 \mathrm{~cm}$, while the value of $\sigma_{c X}^{\max }$ is equal to $1.51 \mathrm{~cm}$; afterwards $\gamma=9$. This result leads to several considerations. First of all, $\sigma_{c X}^{\max }$ is determined performing a probabilistic analysis based on the consideration that the structural data are deterministic, and so assuming that the knowledge of the parameters is complete. Instead, if the parameters are known with a certain degree of uncertainty, a mean estimate of the response is given by the centroid value which is one order greater than the nominal value. However, it has to be underlined that this evaluation corresponds to a low degree of membership which rarely occurs in the practical applications. Then the coefficient $\gamma$ is a measure of how much the probabilistic response value is amplified if an average degree of informal uncertainty is assumed in the values of the parameters.

\section{CONCLUSIONS}

The proposed approach has been adopted to evaluate the structural response of a linear TMD subject to an earthquake input modelled as the Kanai and Tajimi filtered nonstationary process. In the space state, the dynamical analysis has been conducted considering that all the involved structural and filter parameters are fuzzy sets; this choice has been done to consider that in real analyses all of them are affected by a 


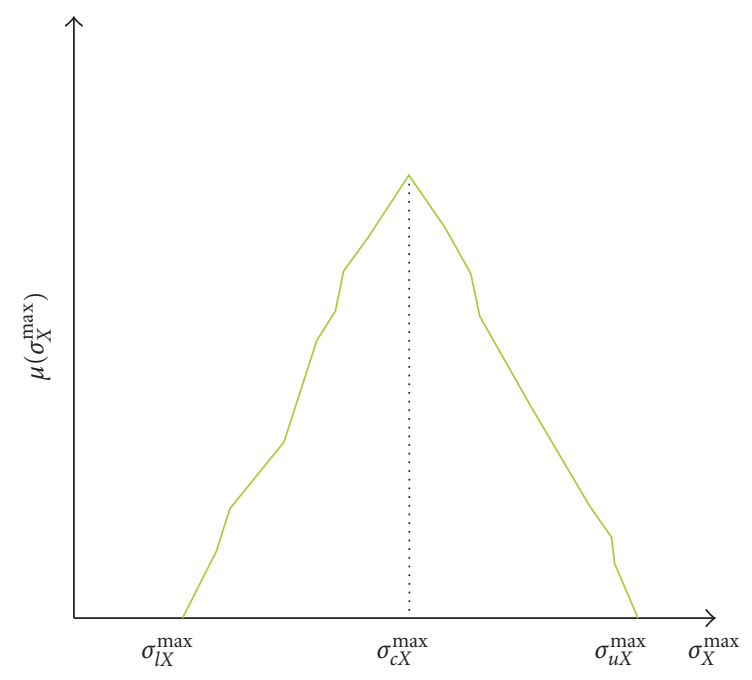

FIgURE 7: Generic representation of a membership function with representation of symbols used in (23).

degree of uncertainty which cannot be quantified only using a probabilistic approach. In fact, it is necessary to take into account the uncertainties also. The fuzzy structural analysis has been performed by means of the $\alpha$-optmization method. A genetic algorithm has been used to gain the optimal point which is able to define the output MF under the hypothesis that the MFs of the input variables are all triangular. The results show the effectiveness of the numerical proposed procedure, demonstrating that assuming a high-medium level of confidence and considering that the core of the output MF corresponds to the probabilistic mean value, the estimation of the structural response is almost the same if the purely probabilistic structural analysis is performed. Afterwards, a sensitivity analysis in respect to the structural frequency and damping ratio has been conducted. The results of this analysis show that a scattering in $\widetilde{\omega}_{s}$ has more influence in the output MF. So, judging inopportunely these parameters without considering in a proper way the degree of uncertainty as using wrong values for them could induce an erroneous assessment of the structural response. Finally, a structural amplification factor is obtained by dividing the centroid value of a fuzzy output number and of the core one. When the amplification factor is greater than one, the structural response is overrated regarding the stochastic approach. This clearly shows that the fact of performing a stochastic analysis of a structural system and of considering a certain degree of uncertainty on its parameters is often not sufficient to properly evaluate the structural response and this could induce an erroneous assessment of its values. Frequently, this scattering can be also very huge, so the analysis process cannot be properly controlled.

\section{REFERENCES}

[1] T. T. Soong and B. F. Spencer Jr., "Supplemental energy dissipation: state-of-the-art and state-of-the-practice," Engineering Structures, vol. 24, no. 3, pp. 243-259, 2002.
[2] F. Rüdinger, "Optimal vibration absorber with nonlinear viscous power law damping and white noise excitation," Journal of Engineering Mechanics, vol. 132, no. 1, pp. 46-53, 2006.

[3] G. C. Marano, R. Greco, F. Trentadue, and B. Chiaia, "Constrained reliability-based optimization of linear tuned mass dampers for seismic control," International Journal of Solids and Structures, vol. 44, no. 22-23, pp. 7370-7388, 2007.

[4] G. C. Marano, R. Greco, and G. Palombella, "Stochastic optimum design of linear tuned mass dampers for seismic protection of high towers," Structural Engineering and Mechanics, vol. 29, no. 6, pp. 603-622, 2008.

[5] G. C. Marano, S. Sgobba, R. Greco, and M. Mezzina, "Robust optimum design of tuned mass dampers devices in random vibrations mitigation," Journal of Sound and Vibration, vol. 313, no. 3-5, pp. 472-492, 2008.

[6] M.-Y. Liu, W.-L. Chiang, J.-H. Hwang, and C.-R. Chu, "Windinduced vibration of high-rise building with tuned mass damper including soil-structure interaction," Journal of Wind Engineering and Industrial Aerodynamics, vol. 96, no. 6-7, pp. 1092-1102, 2008.

[7] M. Setareh, J. K. Ritchey, T. M. Murray, J.-H. Koo, and M. Ahmadian, "Semiactive tuned mass damper for floor vibration control," Journal of Structural Engineering, vol. 133, no. 2, pp. 242-250, 2007.

[8] I. Takewaki and Y. Ben-Haim, "Info-gap robust design with load and model uncertainties," Journal of Sound and Vibration, vol. 288, no. 3, pp. 551-570, 2005.

[9] L. A. Zadeh, "Fuzzy sets," Information and Control, vol. 8, no. 3, pp. 338-353, 1965.

[10] C.-W. Chen, K. Yeh, W.-L. Chiang, C.-Y. Chen, and D.J. Wu, "Modeling, $\mathrm{H}_{\infty}$ control and stability analysis for structural systems using Takagi-Sugeno fuzzy model," Journal of Vibration and Control, vol. 13, no. 1, pp. 1519-1534, 2007.

[11] F.-H. Hsiao, C.-W. Chen, Y.-W. Liang, S.-D. Xu, and W.-L. Chiang, "T-S fuzzy controllers for nonlinear interconnected systems with multiple time delays," IEEE Transactions on Circuits and Systems I, vol. 52, no. 9, pp. 1883-1893, 2005.

[12] F.-H. Hsiao, C.-W. Chen, Y.-H. Wu, and W.-L. Chiang, "Fuzzy controllers for nonlinear interconnected TMD systems with external force," Journal of the Chinese Institute of Engineers, vol. 28, no. 1, pp. 175-181, 2005.

[13] M. Savoia, "Structural reliability analysis through fuzzy number approach, with application to stability," Computers \& Structures, vol. 80, no. 12, pp. 1087-1102, 2002.

[14] B. Möller, M. Beer, W. Graf, and J.-U. Sickert, "Timedependent reliability of textile-strengthened RC structures under consideration of fuzzy randomness," Computers \& Structures, vol. 84, no. 8-9, pp. 585-603, 2006.

[15] L. Hu, R. Wu, and S. Shao, "Analysis of dynamical systems whose inputs are fuzzy stochastic processes," Fuzzy Sets and Systems, vol. 129, no. 1, pp. 111-118, 2002.

[16] B. Möller, W. Graf, and M. Beer, "Fuzzy structural analysis using $\alpha$-level optimization," Computational Mechanics, vol. 26, no. 6, pp. 547-565, 2000.

[17] S. Lignon and L. Jézéquel, "A robust approach for seismic damage assessment," Computers \& Structures, vol. 85, no. 1-2, pp. 4-14, 2007.

[18] MATLAB Standard Optimization Toolbox, User's Manual, 2008.

[19] H. Tajimi, "A statistical method of determining the maximum response of a building during earthquake," in Proceedings of the 2nd World Conference on Earthquake Engineering, vol. 2, pp. 781-798, Tokyo, Japan, July 1960. 
[20] H. A. Buchholdt, Structural Dynamics for Engineers, Thomas Telford, London, UK, 1997.

[21] P. C. Jennings and G. W. Housner, "Simulated earthquake motions for design purpose," in Proceedings of the 4th World Conference on Earthquake Engineering, vol. A-1, pp. 145-160, Santiago, Chile, December 1968.

[22] C.-H. Yeh and Y. K. Wen, "Modeling of nonstationary ground motion and analysis of inelastic structural response," Structural Safety, vol. 8, no. 1-4, pp. 281-298, 1990. 

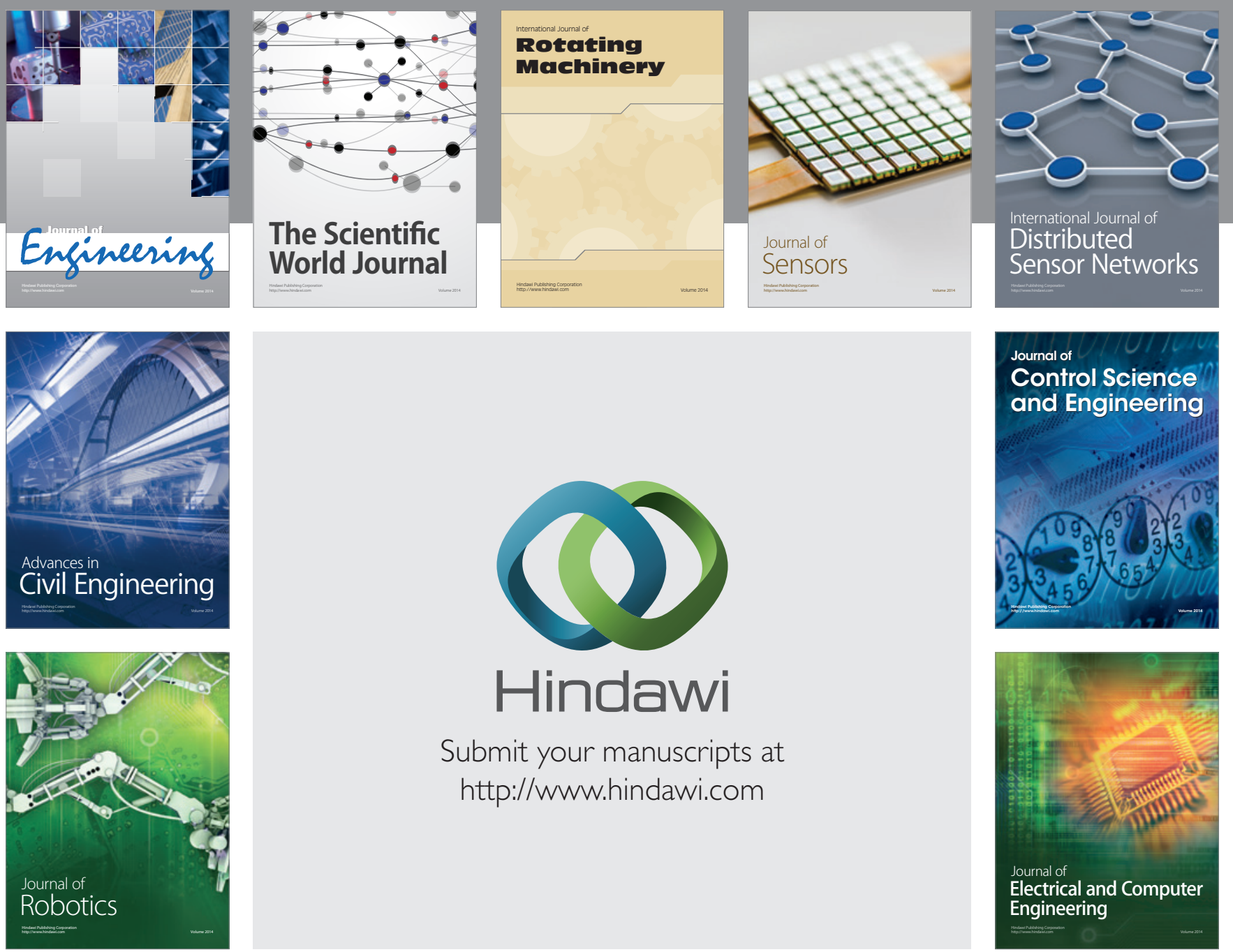

Submit your manuscripts at

http://www.hindawi.com
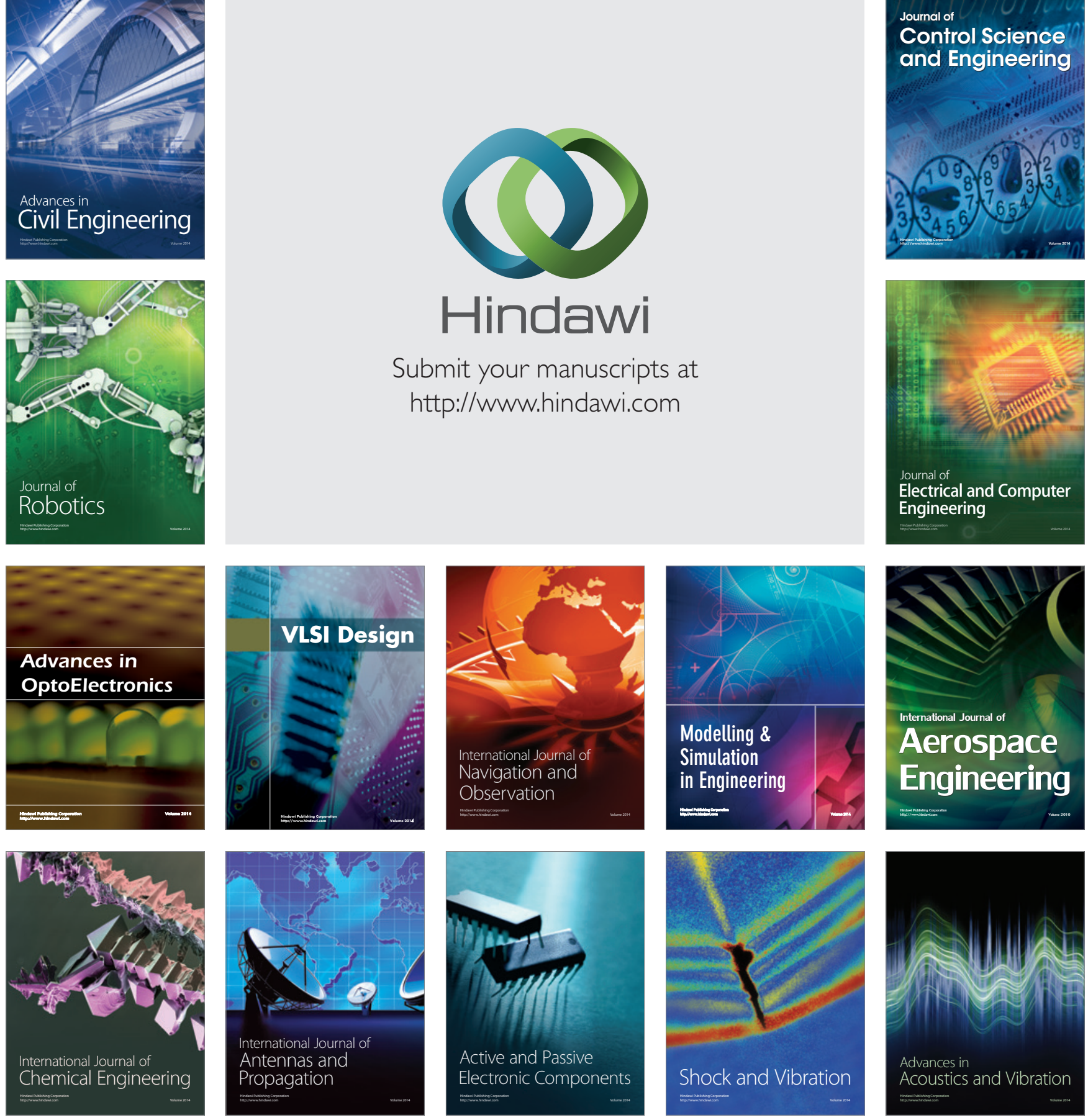